\title{
Efecto hepatoprotector y actividad antioxidante del extracto hidroalcohólico del fruto de dos variedades de Opuntia megacantha "Tuna"
}

\author{
Hepatoprotective effect and antioxidant activity of the hydroalcoholic extract \\ of the fruit of two varieties of Opuntia Megacantha "Tuna"
}

\author{
Edwin C. Enciso ${ }^{1}$, Enrique J. Aguilar ${ }^{2}$, Pablo W. Común ${ }^{2}$, Yovani M. Condorhuamán ${ }^{3}$
}

Recibido: 13/05/2020 Aceptado: 31/07/2020 Publicado: 31/08/2020

\begin{abstract}
RESUMEN
El presente trabajo de investigación se realizó con el objetivo de determinar el efecto hepatoprotector y actividad antioxidante del extracto hidroalcohólico del fruto de dos variedades de Opuntia megacantha "tuna", morada y anaranjada. El contenido de fenoles totales se determinó por el método de Folin-Ciocalteu y los flavonoides por el método del cloruro de aluminio. La actividad antioxidante por los métodos de DPPH, ABTS y FRAP; y el efecto hepatoprotector mediante ensayo in vivo en ratas albinas de 2 meses de edad, con pesos entre $200 \pm 20 \mathrm{~g}$, distribuidas aleatoriamente en siete grupos de 5 animales cada uno, los cuales recibieron: grupo I (suero fisiológico), grupo II ( $\left.\mathrm{CCl}_{4}\right)$, grupo III (silimarina), grupos IV y V (extracto de tuna morada a 250 y $500 \mathrm{mg} / \mathrm{kg}$ ), grupos VI y VII, (extracto de tuna anaranjada a 250 y $500 \mathrm{mg} / \mathrm{kg}$ ). En suero sanguíneo se determinó la actividad de aspartato aminotransferasa (AST) y alanina aminotransferasa (ALT) y se realizó el estudio histopatológico del hígado. La variedad morada presentó mayor contenido de fenoles totales, flavonoides y actividad antioxidante en relación a la variedad anaranjada $(p<0,05)$. La actividad de AST y ALT a la dosis de $250 \mathrm{mg} / \mathrm{kg}$ son estadísticamente similares a la de silimarina para los extractos de tuna morada y anaranjada. En el estudio histopatológico, ambas variedades demostraron protección frente al daño del tetracloruro de carbono. En conclusión, el extracto hidroalcohólico de las variedades de tuna morada y anaranjada demostraron tener efecto hepatoprotector y actividad antioxidante.
\end{abstract}

Palabras clave: Opuntia megacantha; tuna; hepatoprotector; actividad antioxidante.

\section{SUMMARY}

The present research work was carried out with the aim of determining the hepatoprotective effect and antioxidant activity of the hydroalcoholic extract of the fruit of two varieties of Opuntia megacantha "tuna", purple and orange. The total phenol content was determined by the Folin-Ciocalteu method and the flavonoids by the aluminum chloride method. Antioxidant activity by the DPPH, ABTS and FRAP methods; and the hepatoprotective effect by means of an in vivo test in 2-month-old albino rats, weighing between $200 \pm 20 \mathrm{~g}$, randomly distributed in seven groups of 5 animals each, which received: group I (physiological serum), group II ( $\mathrm{CCl}_{4}$ ), group III (silymarin), groups IV and V (extract of purple prickly pear at 250 and 500 $\mathrm{mg} / \mathrm{kg}$ ), groups VI and VII, (extract of orange prickly pear at 250 and $500 \mathrm{mg} / \mathrm{kg}$ ). In blood serum, the activity of aspartate aminotransferase (AST) and alanine aminotransferase (ALT) was determined and the histopathological study of the liver was performed. The purple variety had a higher content of total phenols, flavonoids and antioxidant activity in relation to the orange variety $(p<0.05)$. The activity of AST and ALT at the dose of $250 \mathrm{mg} / \mathrm{kg}$ are statistically similar to that of silymarin for extracts of purple and orange prickly pear. In the histopathological study, both varieties showed protection against carbon tetrachloride damage. In conclusion, the hydroalcoholic extract of the purple and orange prickly pear varieties were shown to have a hepatoprotective effect and antioxidant activity.

Keywords: Opuntia megacantha; tuna; hepatoprotective; antioxidant activity.

1 Universidad Nacional de San Cristóbal de Huamanga. Facultad de Ciencias de la Salud. Autor para correspondencia: encisogf@hotmail.com

2 Universidad Nacional de San Cristóbal de Huamanga. Facultad de Ciencias de la Salud.

3 Universidad Nacional Mayor de San Marcos. Facultad de Farmacia y Bioquímica. Lima, Perú. E-mail: ycondorhuamanf@unmsm.edu.pe

ORCID: https://orcid.org/0000-0002-6096-865X

\section{Citar como:}

Enciso, E., Aguilar, E., Común, P. y Condorhuamán, Y. (2020). Efecto hepatoprotector y actividad antioxidante del extracto hidroalcohólico del fruto de dos variedades de Opuntia megacantha "Tuna". Ciencia e Investigación 2020 23(1):51-58. doi: http://dx.doi.org/10.15381/ci.v23i1.18752

(c) Los autores. Este artículo es publicado por la Ciencia e Investigación de la Facultad de Farmacia y Bioquímica de la Universidad Nacional Mayor de San Marcos. Este es un artículo de acceso abierto, distribuido bajo los términos de la licencia Creative Commons Atribucion - No Comercia_Compartir Igual 4.0 Internacional. (http://creativecommons.org/licenses/by-nc-sa/4.0/) que permite el uso no comercial, distribución y reproducción en cualquier medio, siempre que la obra original sea debidamente citada. 


\section{INTRODUCCIÓN}

Las enfermedades hepáticas se han convertido en un importante problema de salud pública debido a que el hígado por su función metabólica y desintoxicante es afectado por numerosas sustancias como son: fármacos, toxinas o virus. El daño hepático está asociado con necrosis celular, fibrosis, aumento de la peroxidación y el agotamiento del nivel de glutatión en los tejidos. La mayoría de los químicos hepatotóxicos dañan las células del hígado principalmente al inducir la peroxidación lipídica y otros daños oxidativos en el hígado ${ }^{1}$. Los principales indicadores del daño hepático son las enzimas aspartato aminotransferasa (AST), alanina aminotransferasa (ALT), gamma glutamiltranspeptidasa (GGTP) y fosfatasa alcalina (FA); la bilirrubina, albúmina y actividad de protrombina miden la capacidad funcional del hígado². Los problemas hepáticos causados por los xenobióticos (alcohol) ha llevado a la población a la automedicación y/o al uso de la medicina tradicional, principalmente plantas que contengan antioxidantes ${ }^{3}$.

Los antioxidantes naturales se encuentran en muchas plantas medicinales en forma de ácidos fenólicos, flavonoides, carotenoides que tienen muchas propiedades protectoras frente diversas enfermedades degenerativas. Diversos estudios demuestran que el consumo regular de frutas aportan agentes antioxidantes al organismo, el cual afecta positivamente el equilibrio redox del cuerpo, disminuye el daño oxidativo a los lípidos y mejora el estado antioxidante en las personas ${ }^{4}$; por lo tanto, un aumento en la ingesta de estos antioxidantes dietarios mejorarían la salud. La acción antioxidante que tienen las frutas como la tuna es debido a que constituyen una fuente importante de compuestos, principalmente por la presencia de betalaínas polifenoles, vitaminas, ácidos grasos poliinsaturados y aminoácidos ${ }^{5}$, cuyo consumo regular puede ayudar a prevenir enfermedades degenerativas crónicas, asociado con otros factores dietéticos y estilo de vida ${ }^{6}$.

El género opuntia ha sido ampliamente utilizado en la medicina popular, en alimentos y en el campo farmacéutico por su contenido de vitaminas y minerales ${ }^{7}$, ha demostrado propiedades antioxidantes por su contenido de flavonoides, ácido ascórbico y carotenoide ${ }^{8}$.

La forma silvestre (con espinas) de la tuna, descripta como Opuntia megacantha Salm-Dick, tiene amplia distribución de crecimiento en la región Ayacucho, principalmente en zonas áridas y desérticas, cuyo fruto es consumido como alimento y por sus propiedades medicinales, ya que es rico en compuestos fenólicos ${ }^{9}$. Además la tuna aporta diversos nutrientes ${ }^{\mathbf{1 0}}$, así como altas concentraciones de agentes antioxidantes que le confieren al fruto las características de un alimento medicinal ${ }^{11}$, es decir, prevenir enfermedades, mantener la buena salud y comportarse como hepatoprotector ${ }^{12}$.

En el presente trabajo de investigación se planteó como objetivo general: determinar el efecto hepatoprotector y actividad antioxidante del extracto hidroalcohólico del fruto de dos variedades de Opuntia megacantha "tuna".

\section{MATERIALES Y MÉTODOS}

\section{Tipo de investigación}

La investigación es de tipo experimental con manipulación del factor de estudio y aleatorización del grupo experimental y grupo control para determinar el efecto hepatoprotector del fruto de Opuntia megacantha "tuna" de las variedades morada y anaranjada, recolectadas en la localidad de Alanya, distrito de Tambillo, departamento de Ayacucho. Posteriormente, las muestras fueron analizadas en los laboratorios de Farmacia de la Universidad Nacional de San Cristóbal de Huamanga.

\section{Obtención del extracto hidroalcohólico del fruto de la tuna}

A los frutos de cada una de las variedades se les retiró la cáscara y luego fueron desmenuzados y macerados en etanol de $96 \%$ por siete días con agitación periódica en la proporción de $2000 \mathrm{~g}$ con 4 litros de etanol. Se filtró y se volvió a macerar el residuo hasta agotamiento con etanol de $96 \%$ (1:5) por siete días, se juntaron los extractos, se concentró y secó a $45^{\circ} \mathrm{C}$ en baño María y estufa.

\section{Ensayo fitoquímico del extracto}

Se realizaron ensayos de coloración y precipitación siguiendo el método descrito por Lock de Ugaz ${ }^{13}$.

\section{Determinación del contenido de fenoles totales}

Fue determinado utilizando el reactivo Folin-Ciocalteu (Merck), siguiendo el método descrito por Thangaraj ${ }^{14}$. Se mezcló $50 \mu \mathrm{L}$ de los extractos obtenidos con $0,5 \mathrm{~mL}$ del reactivo de Folin-Ciocalteu $1 \mathrm{~N}$ y $2,5 \mathrm{~mL}$ de solución de carbonato de sodio al $5 \%$. La mezcla fue incubada en la oscuridad por 40 minutos a temperatura ambiente $\left(20^{\circ} \mathrm{C}\right)$. Después de la incubación, la absorbancia fue medida a $725 \mathrm{~nm}$ utilizando un espectrofotómetro visible Thermo Scientific Genesys 10. Se preparó una curva de calibración con ácido gálico a partir de una solución de trabajo de $50 \mu \mathrm{g} / \mathrm{mL}(0,2: 0,4 ; 0,6 ; 0,8$ y 1,0 $\mathrm{mL}$ ). Los resultados fueron expresados en mg equivalentes a ácido gálico por g de extracto hidroalcohólico (mg EAG/g de extracto).

\section{Determinación del contenido de flavonoides}

Se determinó utilizando el método descrito por Thangaraj ${ }^{14}$. Una alicuota de $0,50 \mathrm{~mL}$ del extracto se mezcló con $0,50 \mathrm{~mL}$ de agua destilada y $0,15 \mathrm{~mL}$ de solución de nitrito de sodio al $5 \%$ en un tubo de ensayo. Después de 5 minutos se adicionó $0,15 \mathrm{~mL}$ de cloruro de aluminio al $10 \%$. A los 6 minutos, $2,0 \mathrm{~mL}$ de hidróxido de sodio al $4 \%$ fue ańadido a la mezcla. Inmediatamente, la solución fue completada hasta $5,0 \mathrm{~mL}$ con agua destilada y completamente mezclada. La absorbancia de la mezcla final fue determinada a $510 \mathrm{~nm}$ contra un blanco de la reacción. Se preparó una curva de calibración con quercetina a partir de una solución de trabajo de $200 \mu \mathrm{g} / \mathrm{mL}(0,2: 0,4 ; 0,6 ; 0,8 ; 1,0 \mathrm{~mL})$. El contenido de flavonoides de los extractos fue expresado como mg equivalentes a quercetina/g de extracto (mg EQ/g). 
Determinación de la actividad antioxidante por el método de secuestramiento del radical libre 1,1 - difenil - picril - hidrazilo (DPPH)

Se utilizó el método descrito por Carciochi et al. ${ }^{15}$. A una alícuota del extracto $(150 \mu \mathrm{L})$ de extracto se adicionó a $2850 \mu \mathrm{L}$ de una solución metanólica del radical libre DPPH $(20 \mathrm{mg} / \mathrm{L})$ con una absorbancia ajustada a 1,1 $\pm 0,02 \mathrm{~nm}$ leída a $517 \mathrm{~nm}$. Después de la agitación, la mezcla fue incubada en la oscuridad por 30 minutos y la absorbancia medida a 517 nm en un espectrofotómetro visible Thermo Scientific Genesys 10. Se preparó una curva estándar con Trolox (25 - $800 \mu \mathrm{mol})$. Los resultados son expresados como $\mu$ moles equivalentes a Trolox por gramo de muestra ( $\mu$ mol ET/g de muestra).

Determinación de la actividad antioxidante por el método de secuestramiento del catión radical del ácido $2,2^{\prime}$ - azinobis - (3 - etilbenzotiazolina) - 6 - sulfónico $\left(\mathrm{ABTS}^{+}\right)$

Se utilizó el procedimiento descrito por Thaipong et al. ${ }^{16}$. Se preparó una solución patrón (SP) constituida por 7,4 mM de ABTS y 2,6 mM de persulfato de potasio a los cuales se dejó que reaccionen por 12 horas. La solución de trabajo (ST) fue preparada a partir de $1 \mathrm{~mL}$ de SP disuelto en metanol y se ajustó la absorbancia a $1,1 \pm 0,02 \mathrm{~mL}$, diluyendo con metanol a una longitud de onda de $734 \mathrm{~nm}$. La muestra $(150 \mu \mathrm{L})$ fue mezclada con $2850 \mu \mathrm{L}$ de solución de ABTS y se dejó que reaccionen en la oscuridad por 2 horas y se leyó la absorbancia a $734 \mathrm{~nm}$. Se preparó una curva estándar con Trolox $(25-600 \mu \mathrm{mol})$. Los resultados son expresados como mmol equivalentes de Trolox/g de muestra (mmol ET/g de muestra).

Determinación de la actividad antioxidante por el método de reducción de hierro (FRAP)

Se realizó según la técnica descrita por Thangaraj ${ }^{14}$. El reactivo FRAB se preparó con buffer acetato $\mathrm{pH} 3,6$; $20 \mathrm{mM}$ de TPTZ disueltos en $\mathrm{HCl} 40 \mathrm{mM}$ y $20 \mathrm{mM}$ de cloruro férrico hexahidratado. La solución de trabajo (ST) se obtuvo mezclando y calentando a $37^{\circ} \mathrm{C}, 25 \mathrm{~mL}$ de buffer acetato con 2,5 mL de solución de TPTZ y 2,5 $\mathrm{mL}$ de la solución de $\mathrm{FeCl}_{3}$ Se mezcló $150 \mu \mathrm{L}$ de muestra con $2850 \mu \mathrm{L}$ de solución ST, dejándose reaccionar por 30 minutos y se leyó la absorbancia a $593 \mathrm{~nm}$. Se preparó una curva estándar con Trolox $(25-800 \mu \mathrm{M})$. Los resultados fueron expresados como mmol equivalentes de Trolox/g de extracto (mmol ET/g de extracto).

\section{Evaluación del efecto hepatoprotector in vivo}

Se utilizó 35 ratas albinas Holtzman, de 2 meses de edad, con pesos comprendidos entre $200 \pm 20 \mathrm{~g}$, distribuidas en 7 grupos de 5 animales cada uno, agrupados en jaulas individuales, en el bioterio de la Universidad Nacional de San Cristóbal de Huamanga, con control de temperatura y horas de luz y oscuridad. Una semana antes de iniciar propiamente el experimento, las ratas solo recibieron una dieta normocalórica, normoproteica y agua ad libitum. Los tratamientos se realizaron por vía orogástrica de la siguiente forma: Grupo I (suero fisiológico); Grupo II ( $\left.\mathrm{CCl}_{4}\right)$ se le administró suero fisiológico durante 7 días y $\mathrm{CCl}_{4}(1 \mathrm{~mL} / \mathrm{kg}$ de peso i.p. al 50 $\%$ v/v con aceite de oliva) en el $6^{\circ}$ y $7^{\circ}$ día; Grupo III (silimarina $100 \mathrm{mg} / \mathrm{kg}$ de peso/d) y $\mathrm{CCl}_{4}$; los grupos de estudio IV y $\mathrm{V}$, recibieron el extracto de tuna morada (250 y $500 \mathrm{mg} / \mathrm{kg}$ de peso/d) respectivamente, y $\mathrm{CCl}_{4} \mathrm{y}$ los grupos de estudio VI y VII, a los cuales se les administró el extracto de tuna anaranjada $(250$ y $500 \mathrm{mg} / \mathrm{kg}$ de peso/d) respectivamente, y $\mathrm{CCl}_{4}{ }_{4}^{17}$. $\mathrm{Al} 8^{\circ}$ día todos los animales fueron anestesiados y se les practicó eutanasia por administración de tiopental por vía $\mathrm{IP}^{18}$. Se recolectó sangre del corazón, la cual fue centrifugada a 3500 rpm por $15 \mathrm{~min}$, para la obtención de suero y realizar los análisis bioquímicos. La muestra de hígado fue conservada en formalina al $10 \%$ para su estudio histopatológico ${ }^{19}$. El cuidado de los animales de experimentación fue realizado de acuerdo con los lineamientos dados por el Instituto Nacional de Salud ${ }^{20}$ y a la declaración de la Asociación Médica Mundial ${ }^{21}$.

La determinación de la actividad de AST y ALT se realizó empleando los reactivos de la casa Wiener Lab ${ }^{22}$, para lo cual en tubos marcados con B (blanco) y T (tratamiento) se agregaron el sustrato para AST y ALT y se colocaron en baño de agua a $37^{\circ} \mathrm{C}$ por 5 minutos, luego se agregó $100 \mu \mathrm{L}$ de agua al tubo B, y $100 \mu \mathrm{L}$ de suero al tubo $\mathrm{T}$ según tratamiento, los cuales se mezclaron por agitación y se incubaron 30 minutos. Se agregaron a cada tubo $0,5 \mathrm{~mL}$ de 2,4-dinitrofenilhidracina $1 \mathrm{mM}$, se mezclaron y se dejaron 10 minutos a $37^{\circ} \mathrm{C}$, luego $5 \mathrm{~mL}$ de hidróxido de sodio $0,4 \mathrm{M}$, se mezclaron por inversión y realizándose la lectura a $505 \mathrm{~nm}$. Para el cálculo de valores se emplearon curvas de calibración. Para la evaluación histopatológica del hígado se realizaron cortes de 3 a $5 \mu \mathrm{m}$ de espesor y se colorearon con hematoxilina y eosina ${ }^{19}$.

\section{Análisis de datos}

Los datos obtenidos se presentan como medias \pm desviación estándar y se representan en tablas y figuras. Las diferencias entre las medias se analizaron mediante la prueba T-Student para el contenido de fenoles totales, flavonoides y actividad antioxidante y análisis de varianza para evaluar la actividad de las transaminasas AST y ALT y la prueba de comparaciones múltiples de Tukey y Dunnett con un nivel de confianza del $95 \%$, para el cual se utilizó el paquete estadístico SPSS versión 20.

\section{RESULTADOS}

En el extracto hidroalcohólico del fruto de las variedades de tuna morada y anaranjada, se encontraron la presencia de compuestos fenólicos, taninos, flavonoides, azúcares reductores, catequinas y lactonas. Los fenoles dieron una coloración verde oscura al ensayo de cloruro férrico; los taninos precipitaron al ensayo de gelatina; los flavonoides una coloración naranja rojizo al ensayo de Shinoda; los azúcares formaron precipitado de color rojo al prueba de Benedic; las catequinas una coloración verde carmelita a la luz ultra violeta, y las lactonas una 
coloración roja al ensayo de Baljet; estos hallazgos concuerdan para el mayor número de metabolitos secundarios presentes en el género opuntia ${ }^{23}$.

En la tabla 1 se observa que el contenido de fenoles totales y flavonoides fue mayor en la variedad morada que en la anaranjada $(\mathrm{p}<0,05)$.

El fruto de la tuna de la variedad morada presenta mayor actividad antioxidante que la variedad anaranjada (Tabla 2), los cuales fueron demostrados con la capacidad de captar los radicales libres DPPH y ABTS y el poder reductor del hierro (FRAB).

En las figuras 1 y 2 se muestran las actividades del aspartato aminotransferasa (AST) y alanina aminotransferasa (ALT) frente al tetracloruro de carbono $\left(\mathrm{CCl}_{4}\right)$. En ambos casos se observan un incremento de la actividad de dichas enzimas en comparación con los grupos que recibieron los tratamientos $(\mathrm{p}<0,05)$. El efecto hepatoprotector de los extractos de la tuna morada y anaranjada a la dosis de $250 \mathrm{mg} / \mathrm{kg}$, es estadísticamente similar a la de silimarina para AST y a la dosis de $250 \mathrm{mg} / \mathrm{kg}$ para tuna morada y $500 \mathrm{mg} / \mathrm{kg}$ para la tuna anaranjada para ALT según la prueba de Tukey.

\section{DISCUSIÓN}

La actividad antioxidante encontrada en ambas variedades de tuna, es por su contenido de fenoles y flavonoides, lo cual está en relación con el trabajo realizado en el fruto de Opuntia ficus indica, donde se reporta la actividad antioxidante por su contenido de vitamina $\mathrm{C}$ y polifenoles ${ }^{24}$.

El fruto de la tuna presenta actividad antioxidante debido a que en la porción comestible contiene abundantes polifenoles y flavonoides que poseen efectos protectores para la salud. Los polifenoles son sustancias naturales que son antioxidantes con el potencial de proteger y combatir enfermedades degenerativas en el ser humano, debido a estos compuestos contiene gran número de grupos hidroxilos con propiedades quelantes ${ }^{25}$.

En trabajos similares, se reportó que la tuna de la variedad morada (púrpura) mostró un contenido muy alto de betacianinas, fenoles, vitamina $\mathrm{C}$ y mayor capacidad antioxidante que la variedad naranja ${ }^{26}$.

Al evaluar el efecto hepatoprotector del extracto hidroalcohólico de tuna, tanto de las variedades morada y anaranjada, se observan protección del hígado en forma similar al grupo tratado con silimarina, al no presentar niveles elevados de la actividad de transaminasas AST y ALT; así como, infiltrado inflamatorio crónico en espacio porta, como se observa en el grupo tratado con tetracloruro de carbono.

$\mathrm{El} \mathrm{CCl}_{4}$ forma radical triclorometilo que causa daño a nivel del hígado, generando necrosis y daño hepático en general $^{27}$.

Los radicales libres están involucrados en daños al hígado, corazón y cáncer ${ }^{28}$. El estrés oxidativo es el principal mecanismo del dańo hepático generado por el tetracloruro de carbono ${ }^{27}$. La terapia antioxidante inhibe los cambios oxidativos deletéreos y es considerada una herramienta muy importante para los tratamientos de las enfermedades hepáticas ${ }^{29}$.

Los frutos de la tuna son consideradas una fuente importante de compuestos antioxidantes por su contenido de fenoles y flavonoides con propiedades hepatoprotectoras, como se demostró al flavonoide astilbina ${ }^{30}$.

La silimarina se viene utilizando en problemas del hígado por sus excelentes propiedades regeneradora y antioxidante ${ }^{31,32}$, neutraliza los radicales libres, estimula la producción de glutatión reducido y mejora el metabolismo hepático ${ }^{33}$.

Según la prueba de Dunnnet, el tratamiento con los extractos del fruto de tuna morada, anaranjada y silimarina más tetracloruro de carbono redujeron el incremento de los marcadores enzimáticos de la hepatotoxicidad (AST y ALT) de forma significativa $(\mathrm{p}<0,05)$, respecto al grupo que sólo recibió $\mathrm{CCl}_{4}$. Estos resultados también fueron reportados en otros estudios publicados, que evidencian el efecto protector contra la administración de tetracloruro de carbono $\left(\mathrm{CCl}_{4}\right)$ más silimarina a $25 \mathrm{mg} / \mathrm{kg}^{31}$.

Tabla 1. Contenido de fenoles totales y flavonoides del extracto hidroalcohólico del fruto de Opuntia megacantha "tuna", variedades morada y anaranjada.

\begin{tabular}{cccc}
\hline Ensayo & $\begin{array}{c}\text { Tuna morada } \\
\text { Media } \pm \mathrm{DE}\end{array}$ & $\begin{array}{c}\text { Tuna anaranjada } \\
\text { Media } \pm \mathrm{DE}\end{array}$ & $\mathrm{p}<$ \\
\hline Fenoles totales (mg EAG/g extracto) & $18,40 \pm 0,09$ & $13,51 \pm 0,09$ & 0,05 \\
Flavonoides (mg EQ/g extracto) & $4,86 \pm 0,15$ & $2,52 \pm 0,25$ & 0,05 \\
\hline
\end{tabular}

Tabla 2. Capacidad secuestradora del radical libre DPPH, ABTS Y FRAB del extracto hidroalcohólico del fruto de Opuntia megacantha "tuna", variedades morada y anaranjada.

\begin{tabular}{clll}
\hline $\begin{array}{c}\text { Ensayo } \\
\text { (mmol ET/g extracto) }\end{array}$ & $\begin{array}{c}\text { Tuna morada } \\
\text { Media } \pm \text { DE }\end{array}$ & $\begin{array}{c}\text { Tuna anaranjada } \\
\text { Media } \pm \text { DE }\end{array}$ & 0 \\
\hline DPPH & $21,46 \pm 0,83$ & $11,68 \pm 1,55$ \\
ABTS & $56,29 \pm 1,10$ & $52,75 \pm 0,32$ \\
FRAB & $52,03 \pm 0,85$ & $36,17 \pm 1,37$ & 0,05 \\
\hline
\end{tabular}




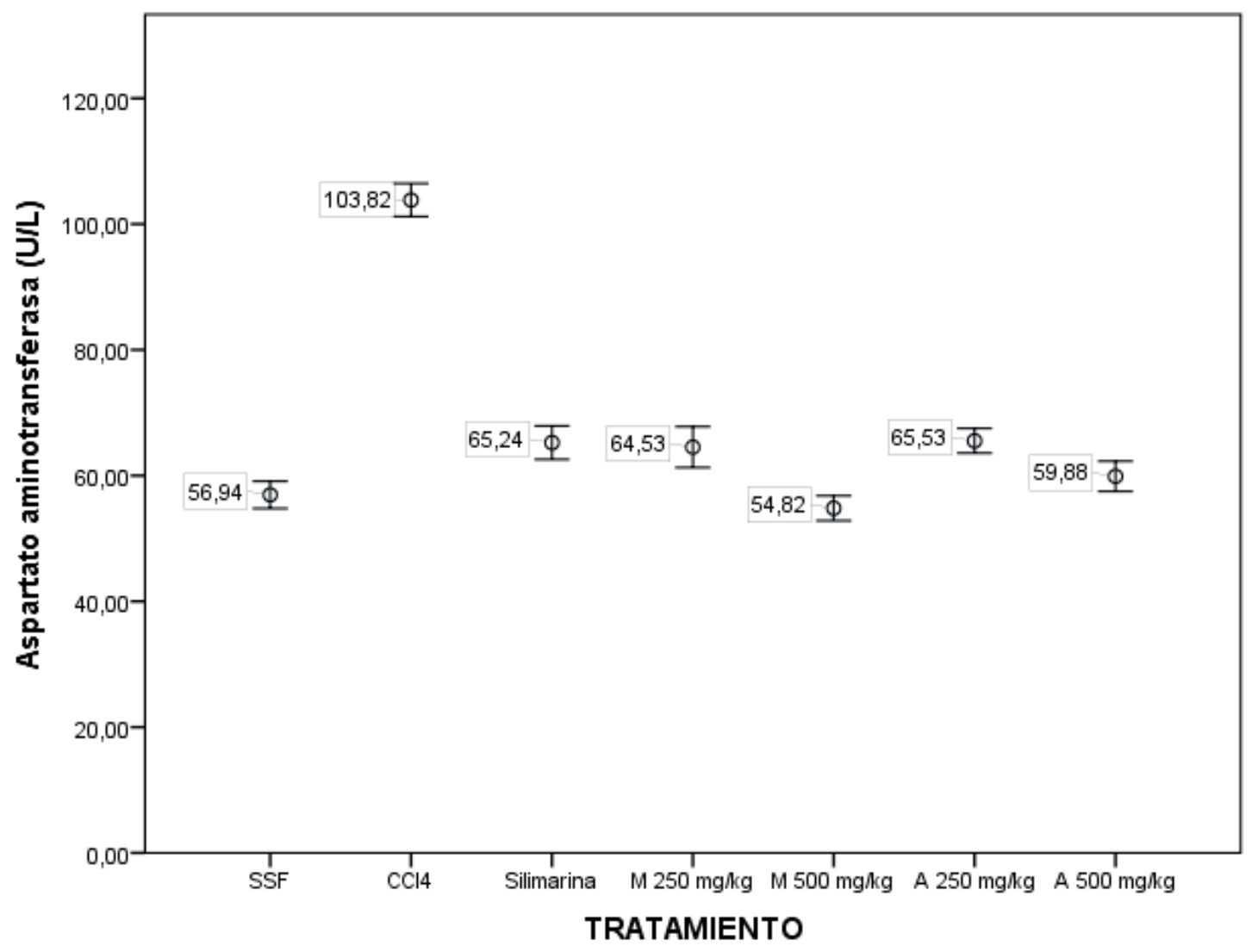

Figura 1. Actividad de aspartato aminotransferasa sérica en ratas al administrar el extracto hidroalcohólico del fruto de Opuntia megacantha "tuna", variedades morada y anaranjada. $p<0,05$.

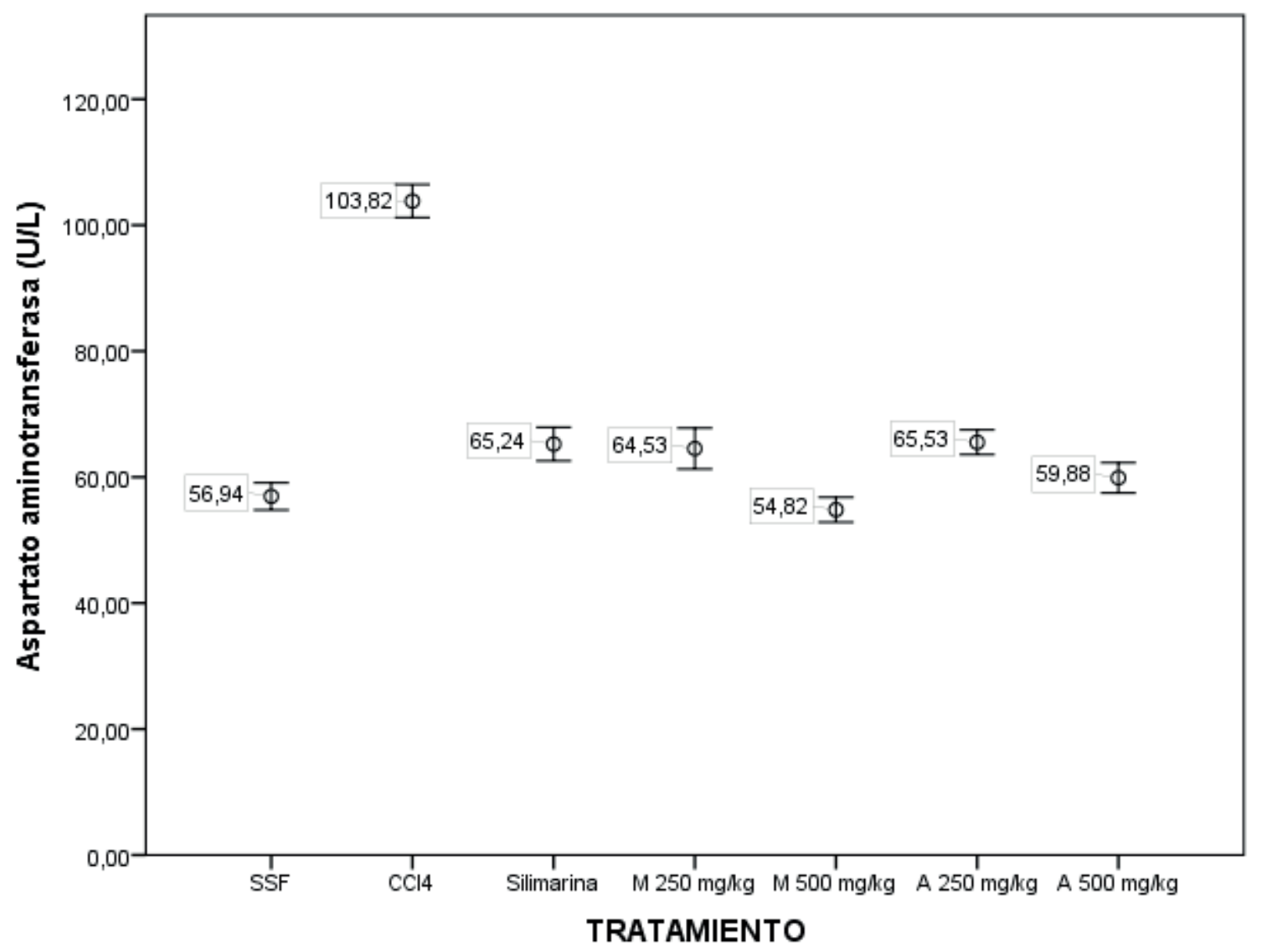

Figura 2. Actividad de alanina aminotransferasa sérica en ratas al administrar el extracto hidroalcohólico del fruto de Opuntia megacantha "tuna", variedades morada y anaranjada. $p<0,05$ 


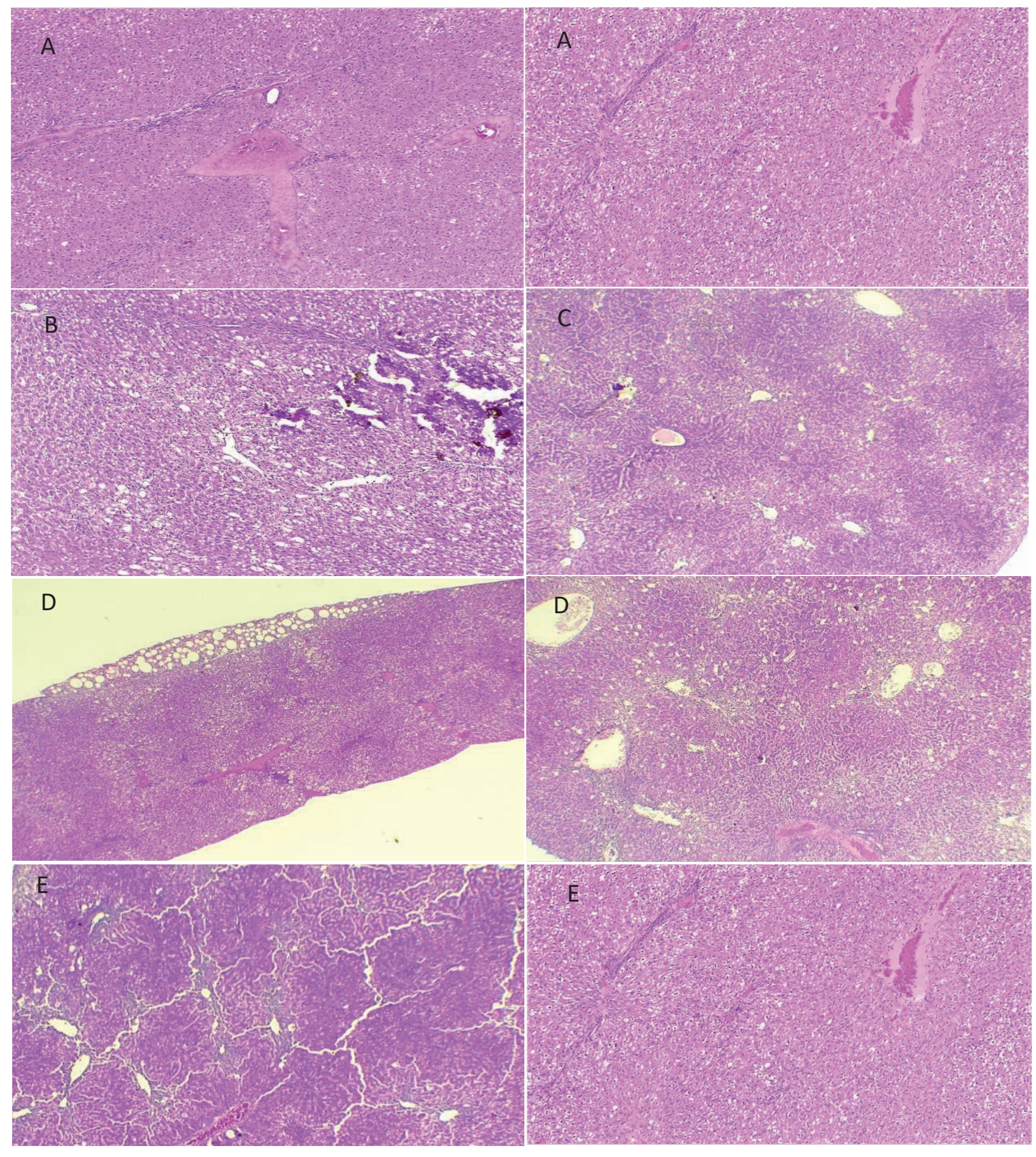

\begin{tabular}{llll}
\hline Grupo & Parénquima & $\begin{array}{l}\text { Infiltrado } \\
\text { inflamatorio }\end{array}$ & Esteatosis \\
\hline A: Control & Homogéneo & Escaso & Ausente \\
B: Silimarina & Homogéneo & Ausente & A gota grande \\
$\mathrm{C}: \mathrm{CCl}_{4}$ & Leve tendencia nodular & Crónico & A gota grande \\
$\mathrm{D}:$ Extracto de tuna morada & Homogéneo & Escaso & Agota grande \\
E: Extracto de tuna anaranjada & Leve tendencia nodular & Escaso & Ausente \\
\hline
\end{tabular}

Figura 3. Estudio histopatológico del hígado de rata al administrar el extracto hidroalcohólico del fruto de Opuntia megacantha "tuna", variedades morada y anaranjada. 
Los resultados del efecto hepatoprotector obtenidos en los grupos que recibieron extracto hidroalcohólico de tuna de las variedades morada y anaranjada y tetracloruro de carbono, pueden explicarse por sus componentes con actividad antioxidante como los flavonoides y fenoles. El daño hepático es causado principalmente por el estrés oxidativo generado por radicales libres, cuyos atenuadores son los antioxidantes, como: vitaminas, minerales, flavonoides y polifenoles ${ }^{34}$.

En resumen, el fruto de la tuna presenta efecto hepatoprotector debido a su contenido de polifenoles, principalmente flavonoides, que son poderosos antioxidantes al disminuir el estrés oxidativo y atrapar radicales libres, tanto in vivo como in vitro; ejerciendo un efecto similar al de la silimarina.

\section{CONCLUSIÓN}

El extracto hidroalcohólico del fruto de Opuntia megacantha "tuna" de las variedades morada y anaranjada presentaron efecto hepatoprotector y actividad antioxidante, siendo superior la variedad morada por el mayor contenido de fenoles totales y flavonoides en relación a la variedad anaranjada.

\section{AGRADECIMIENTOS}

El presente trabajo ha sido realizado gracias al apoyo del Instituto de Investigación de la Facultad de Ciencias de la Salud de la Universidad Nacional de San Cristóbal de Huamanga.

\section{REFERENCIAS BIBLIOGRÁFICAS}

1. Abou Seif H. Physiological changes due to hepatotoxicity and the protective role of some medicinal plants. Beni-Suef University Journal of Basic and Applied Sciences. 2016; 5(2):134-46.

2. Borque MA, Moreno GL, Mendoza-Giménez L, Gracía-Buey L, Otero MR. Utilidad de los parámetros analíticos en el diagnóstico de las enfermedades hepáticas. An. Med. Interna. 2007; 24(1):38-46

3. Osorio L, Patiño T, Tagle M, Huayanay L. Percepciones, conocimientos y actitudes sobre enfermedades hepáticas en adultos sanos que acuden a instituciones de salud de estrato A, B y C. Rev gastroenterol. 2010; 30(21):126-32.

4. Tesoriere L, Butera D, Pintaudi A, Allegra M, Livrea M. Supplementation with cactus pear (Opuntia ficus-indica) fruit decreases oxidative stress in healthy humans: a comparative study with vitamin C. Am J Clin Nutr. 2004; 80:391-5.

5. El-Mostafa K, El Kharrassi Y, Badreddine A, Andreoletti P, Vamecq J, El Kebbaj MS, Latruffe N, Lizard G, Nasser B, Cherkaoui-Malki M. Nopal Cactus (Opuntia ficus-indica) as a Source of Bioactive Compounds for Nutrition, Health and Disease. Molecules. 2014; 19(9):14879-901.

6. López A. Efecto del zumo de mandarina sobre el estrés oxidativo: estudio experimental y en patologías mediadas por radicales libres [Tesis Doctoral]. Valencia - España: Universidad de Valencia; 2014:287.

7. Rodrigo G. Actividad genotóxica de Opuntia soehrensii, evaluada por el test de mutación y recombinación somática en $D$. melanogaster. Biofarbo. 2007; 15(1):61-6.
8. Kuti J. Antioxidant compounds from four Opuntia cactus pear fruit varieties. Food Chem. 2004; 85:527-33.

9. Pascoe-Ortiz S, Rodríguez-Macías R, Robledo-Ortiz JR, Salcedo-Pérez E, Zamora-Natera JF, Rabelero-Velasco M, et al. Identificación de propiedades presentes en jugo de Opuntia megacantha Salm-Dyck importantes para la producción de biopolímeros. Revista especializada en ciencias químico-biológicas. 2019; 22.

10. Sumaya-Martínez MT, Cruz-Jaime S, Madrigal-Santillán JD, García-Paredes R, Cariño-Cortés N, Cruz-Cansino C, et al. Betalain, acid ascorbic, phenolic contents and antioxidant properties of purple, red, yellow and white cactus pears. Int. J. Mol. Sci. 2011; 12:6452-68.

11. Ramírez-Ramos M, García-Mateos MR, Corrales-García J, Ybarra-Moncada C, Castillo-González AM. Compuestos antioxidantes en variedades pigmentadas de tuna (Opuntia sp.). Rev. Fitotec. Mex. 2015; 38(4): 349-57.

12. Sánchez Torres CA, Sotomayor Ríos GC, Huamán Gutiérrez OG. Efecto hepatoprotector del zumo de fruta de Opuntia ficus indica (tuna), variedad morada, en ratas con intoxicación hepática Inducida por paracetamol. Efecto hepatoprotector del zumo de fruta de Opuntia ficus indica (tuna), variedad morada, en ratas con intoxicación hepática inducida por paracetamol. [Tesis]: UNMSM, 2015.

13. Lock de Ugaz. Investigación Fitoquímica. Métodos en el Estudio de los Productos Naturales. Segunda Edición. Fondo Editorial de la Pontifica Universidad Católica del Perú. 1994: 114-33.

14. Thangaraj P. Pharmacological Assays of Plant - Based Natural Products. Series:Progress in Drug Research 71. Editor: K.D. Rainsford Springer. 2016.

15. Carciochi RA, Manrique GD, Dimitrov K. Changes in phenolic composition and antioxidant activity during germination of quinoa seeds (Chenopodium quinoa Willd). International Food Research Journal. 2014;21(2):767-73.

16. Thaipong K, Boonprakob U, Crosby K, Cisneros-Zevallos L, Hawkins Byrne D. Comparison of ABTS, DPPH, FRAP, and ORAC assays for estimating antioxidant activity from guava fruit extracts. Journal of Food Composition and Analysis. 2006; 19, 669-75.

17. Canelo-Saldaña $P$, Mendoza-Gardini $Y$, Villacrés Vallejo J, Aranda-Ventura J, González G. Análisis fitoquímico, actividad antioxidante y hepatoprotector del extracto acuoso liofilizado de Curcuma longa en lesiones hepáticas inducidas con tetraclorometano, en ratas albinas. Rev Peru Med Integrativa. 2017;2(3):765-72.

18. Canadian Council on Animal Care. CCAC guidelines on: euthanasia of animals used in science. Ottawa: Canadian Council on Animal Care; 2010.

19. Arka G, Anindita K, Ankit S, Kumar SA, Kumar MS. Preliminary evaluation of hepatoprotective potential of the polyherbal formulation. J Intercult Ethnopharmacol. 2015;4(2):118-24.

20. Fuentes Paredes FDM, Mendoza Yanavilca RA, RosalesFernández AL, Cisneros Tarmeño RA. Guía de manejo y cuidado de animales de laboratorio: ratón. Instituto Nacional de Salud. Lima, 2008.

21. Asociación médica mundial. Declaración de la AMM sobre el Uso de Animales en la Investigación Biomédica, 2016. 
22. Troncoso L, Guija E. Efecto antioxidante y hepatoprotector de Petroselinum sativum (perejil) en ratas con intoxicación hepática inducida por paracetamol. An Fac Med. 2007; 68(4):333-43.

23. Tomás Ch, Huamán J, Aguirre R, Bravo M, León J, Guerrero $\mathrm{M}$, Orihuela C, Avilés R, Yanqui E. Estudio químico y fitoquímico de la Opuntia ficus-indica "tuna" y elaboración de un alimento funcional. Rev. Per. Quím. Ing. Quím. 2012; 15(1):70-4.

24. Jorge P, Troncoso L. Capacidad antioxidante del fruto de la Opuntia apurimacensis (ayrampo) y de la Opuntia ficus-indica (tuna). An Fac med. 2016;77(2):105-9.

25. Acker SA, Berg DJ, Tromp MN, Griffioen DH, Bennekom WP, W. J. van der Vijgh WJ, Bast A. Structural aspects of antioxidant activity of flavonoids. Free Radic Biol Med. 1996; 20(3):331- 42.

26. Albano C, Negro C, Tommasi N, Gerardi C, Mita G, Miceli A, De Bellis L, Blando F. Betalains, Phenols and Antioxidant Capacity in Cactus Pear [Opuntia ficus-indica (L.) Mill.] Fruits from Apulia (South Italy) Genotypes. Antioxidants. 2015 4(2):269-80.

27. Gurpreet K, Sarwar AM, Zoobi J, Kaleem J, Mohammed A. Evaluation of antioxidant activity of Cassia seamea flowers. $J$ Ethnopharmacol. 2006; 108:340-348.

28. Seitz HK, Stickel F. Risks factors and mechanism of hepatocarcinogenesis with special emphasis on alcohol and oxidative stress. Biol Chem. 2006; 387:349-360.
29. Yang L, Wang CZ, Ye JZ, Li HT. Hepatoprotective effects of polyphenols from Ginkgo biloba L. leaves on $\mathrm{CCl}_{4}$ - induced hepatotoxicity in rats. Fitoterapia. 2011; 82:834-840.

30. Selema de la Morena G, Martínez Pérez J. Efecto hepatoprotector inducido por el flavonoide astilbina frente a un modelo animal tratado con tetracloruro de carbono. Rev Cubana Plant Med. 1999; 1(4):36-9.

31. Arroyo J, Almora Y, Quino M, Raez E, Martínez J, Buendia J, et al. Efecto protector en Cirrosis Hepática inducida en ratas del extracto etanólico de las hojas de Piper aduncum comparado con Silimarina. An. Fac. Med. 2012; 73(2):85-91.

32. Saller R, Brignoli R, Melzer J, Meier R. An updated systematic review with metaanalysis for the clinical evidence of silymarin. Forsch Komplementmed 2008; 15(1): 9-20.

33. Pradhan S, Girish C. Hepatoprotective herbal drug, slymarin from experimental pharmacology to clinical medicine. Indian J Med. 2006; 124:491-504.

34. Sandoval M, Lazarte K, Arnao I. Hepatoprotección antioxidante de la cáscara y semilla de Vitis vinífera L. (uva). An Fac med. 2008; 69(4):250-259.

Conflicto de intereses: Los autores declaran no tener conflictos de interés.

Fuente de financiamiento: Autofinanciado. 